\title{
INTERNACIONALIZAÇÃO DAS UNIVERSIDADES: ESTUDO SOBRE A PRODUÇÃO CIENTÍFICA
}

\author{
INTERNATIONALIZATION OF UNIVERSITIES: \\ STUDY ON SCIENTIFIC PRODUCTION
}

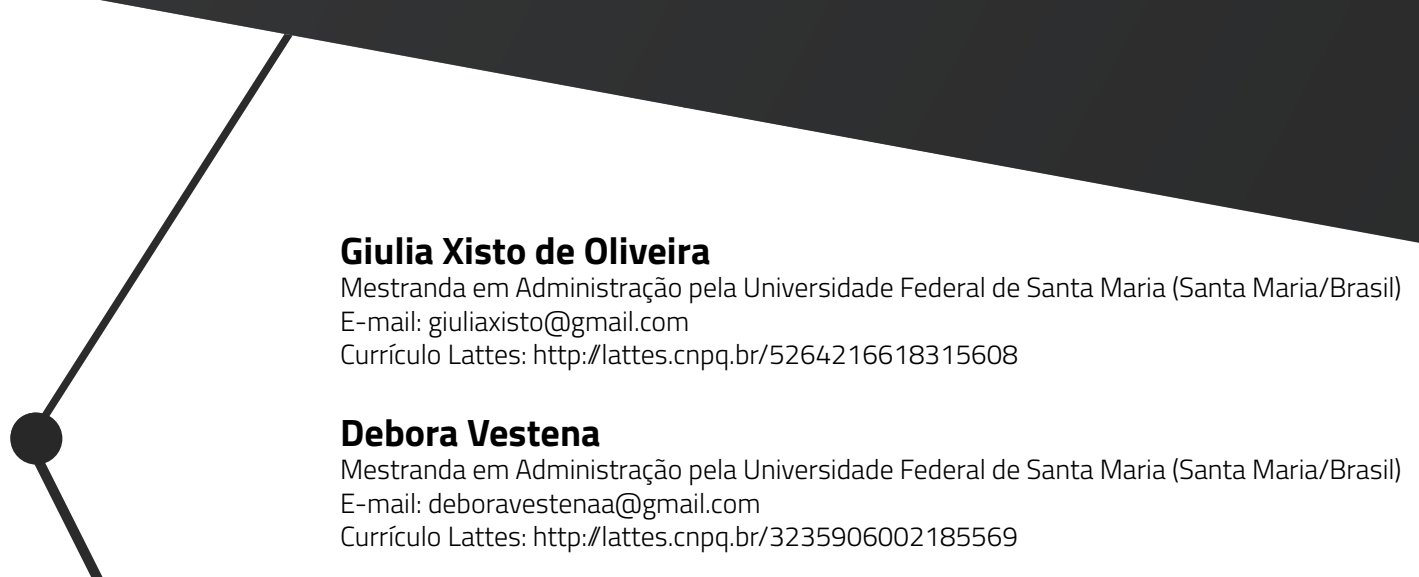

\section{Carlos Rafael Röhrig da Costa}

Doutorando em Administração pela Universidade Federal de Santa Maria (Santa Maria/Brasil)

Servidor Público na Universidade Federal de Santa Maria (Santa Maria/Brasil)

E-mail: crcost@gmail.com

Currículo Lattes: http://lattes.cnpq.br/1568226095968414

\section{Luciana Davi Traverso}

Doutora em Administração pela Universidade Presbiteriana Mackenzie (São Paulo/Brasil)

Docente na Universidade Federal de Santa Maria (Santa Maria/Brasil)

E-mail: luciana.traverso@ufsm.br

Currículo Lattes: http://lattes.cnpq.br/4939993298300369

\section{Roberto Schoproni Bichueti}

Doutor em Administração pela Universidade Federal de Santa Maria (Santa Maria/Brasil)

Docente na Universidade Federal de Santa Maria (Santa Maria/Brasil)

E-mail: roberto.bichueti@ufsm.br

Currículo Lattes: http://lattes.cnpq.br/5060454514705901

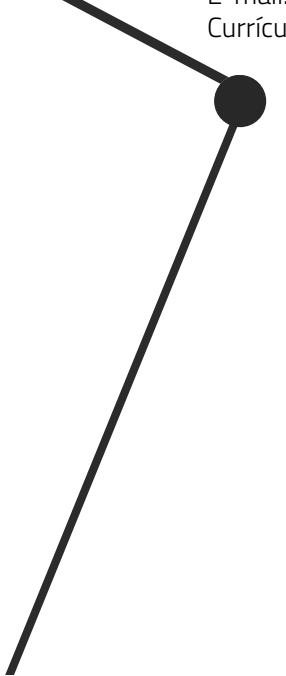

Recebido em: 28 de setembro de 2019

Aprovado em: 25 de novembro de 2019

Sistema de Avaliação: Double Blind Review

RGD | v. 17 |n. 1 | p. 196-217|jan./abr. 2020

DOI: https://doi.org/10.25112/rgd.v17i1.2020 


\section{RESUMO}

A temática internacionalização da educação está cada vez mais presente na realidade das instituições de ensino superior. Essa prática visa a, além de promover a diversidade, possibilitar integrar seu corpo acadêmico à comunidade científica global, favorecendo, assim, a instalação de processos de inovação, competitividade e inserção internacional no país. Neste contexto, o estudo tem como objetivo compreender as características da produção científica internacional referente às temáticas internacionalização e universidade. Para isso, foi desenvolvida uma pesquisa bibliométrica, de natureza descritiva e abordagem quantitativa, utilizando como período de análise os anos de 1989 a 2018. Como principais resultados, destaca-se o crescimento do número de artigos publicados ao longo dos anos, apresentando maior quantidade no ano de 2017. China, Estados Unidos e Inglaterra, respectivamente, apresentam maior número de publicações. Ainda, identificou-se que research, students, development, globalization, science, markets, culture e intercultural experience são possiveis hot topics relacionados à internacionalização e universidade.

Palavras-chave: Internacionalização. Universidades. Bibliometria.

\section{ABSTRACT}

The internationalization theme of education is increasingly present in the reality of higher education institutions. This practice aims, besides promoting diversity, to make it possible to integrate its academic body with the global scientific community, thus favoring the installation of innovation, competitiveness and international insertion processes in the country. In this context, the study aims to understand the characteristics of international scientific production regarding the internationalization and university themes. For this, a descriptive bibliometric research and quantitative approach was developed, using as the analysis period the years 1989 to 2018. As main results, we highlight the growth in the number of articles published over the years, presenting a larger amount. in 2017. China, the United States and England, respectively, have the largest number of publications. Moreover, it was identified that research, students, development, globalization, science, markets, culture and intercultural experience are possible hot topics related to internationalization and university.

Keywords: Internationalization. Universities. Bibliometrics. 


\section{INTRODUÇÃO}

Sabe-se que a globalização impacta fortemente na extensão das atividades econômicas, políticas e sociais e, além disso, funciona de maneira distinta em cada país, pois reúne aspectos culturais, históricos e educacionais (MARTINS; REIS, 2015). Um fator influenciado pelo processo da globalização éa internacionalização de empresas, já que a globalização permite o livre fluxo de bens, serviços, tecnologia e informação entre diferentes organizações e continentes (FINARDI; PORCINO, 2014).

A internacionalização, por sua vez, refere-se à relação existente entre duas ou mais nações, podendo ser de diversos aspectos, como acordos, parcerias, pactos e o mais destacado: as relações financeiras (PUZIOL; BARREYRO, 2016). Para Vieira (2014), internacionalização significa o processo de intensificar o envolvimento de determinada empresa com o mercado internacional, sendo uma das principais estratégias para o desenvolvimento de uma organização, além de trazer diversos desafios geográficos, culturais e econômicos.

Um exemplo da utilização do processo de internacionalização, não tão convencional, são as universidades, que vêm buscando adaptar-se às novas demandas globais de ensino e expandir seus mercados. Segundo Souza, Filipo e Casado (2015), o Brasil apresenta números crescentes no ensino, pesquisa e extensão com cooperação internacional, além disso, a mobilidade entre universidades tem alavancado graças ao Programa Ciência Sem Fronteiras (atualmente denominado Programa Brasileiro de Mobilidade Científica), que busca promover a expansão e internacionalização da ciência e tecnologia, tendo distribuído 78.173 bolsas de intercâmbio para graduação e pós-graduação entre 2011 a 2015.

Porém, sabe-se que a educação superior no Brasil, principalmente na área de internacionalização, tem evoluído lentamente se comparado aos demais países desenvolvidos, já que a limitação do idioma e a falta de incentivos e metodologias adequadas para a inserção internacional do ensino brasileiro ainda são assuntos debatidos (SANTIN; VANZ; STUMPF, 2016). Para os autores, o Brasil precisa integrar políticas públicas e atividades econômicas a fim de internacionalizar a ciência e romper as barreiras atualmente enfrentadas. Já que a internacionalização das universidades contribui para o desenvolvimento de cidadãos e profissionais mais conscientes de outras realidades, com experiências transculturais e preparados para o enfrentamento de desafios globais (MOROSINI; USTARRÓZ, 2016).

Diante da relevância da relação do tema internacionalização no contexto das universidades, verificouse a necessidade de fazer um levantamento das publicações acerca desta temática. Com isso, o objetivo do estudo consiste em verificar as características da produção científica internacional sobre a temática internacionalização na base de dados Web of Science da ISI Web of Knowledge no período de 1989 a 2018.

Assim, o presente estudo está estruturado em cinco capítulos, incluindo esta seção em que são apresentados o objetivo e a relevância do estudo. O segundo capítulo apresenta a contextualização da 
temática Internacionalização e Universidade no âmbito acadêmico, com o propósito de identificar os principais autores e conceitos acerca da temática. O capítulo seguinte apresenta o método do estudo, onde são descritos os procedimentos adotados no desenvolvimento desta pesquisa. Na sequência, é realizada a análise e a discussão dos resultados obtidos e, por fim, as considerações finais.

\section{REFERENCIAL TEÓRICO}

O referencial tem por objetivo fornecer amparo teórico e fundamentar o argumento do estudo, além de dar sustentação posterior discussão e análise dos dados. Assim, o referencial teórico do presente estudo se destina a discutir aspectos relacionados à internacionalização e internacionalização das universidades.

\subsection{INTERNACIONALIZAÇÃO}

De acordo com Vieira (2014), a internacionalização pode ser entendida como um processo pelo qual as organizações intensificam seu envolvimento com o mercado internacional, sendo que o padrão e o ritmo desse processo serão influenciados por fatores econômicos e políticos. 0 autor ainda ressalta que as empresas devem equilibrar os recursos existentes em diversos mercados para capitalizar as imperfeições dos mercados e alavancar seus resultados, por isso, tal expansão geográfica se configura como uma das principais estratégias para o crescimento das empresas.

Dessa forma, diversas teorias buscam explicar o fator da internacionalização, entre elas, pode-se citar a Teoria do Ciclo do Produto (VERNON, 1966), o Processo de Internacionalização da Escola Nórdica de Uppsala (JOHANSON; VAHLNE, 1977), a Distância Psíquica e a Interação Comprador-vendedor (HALLÉN; WIEDERSHEIMPAUL, 1979), Teoria do Paradigma Eclético (DUNNING, 1980), Modelo de Inovação (REID, 1981; CZINKOTA; JOHNSTON, 1985) e Teoria das Operações Internacionais (BUCKLEY; CASSON, 1998).

Na Teoria do Ciclo do Produto de Vernon (1966), a análise parte do princípio de que todos os países desenvolvidos têm acesso ao conhecimento e demais avanços da ciência de forma igualitária e que esses países não aplicam tal conhecimento ao desenvolvimento de novos negócios igualmente, porém, a maioria das empresas busca o desenvolvimento local visando a diminuição de custos com transporte e apenas na fase de maturação do produto é que as empresas avaliam a possibilidade de produção no exterior, com a finalidade de diferenciar-se perante a concorrência e aumentar o ciclo de vida do produto (FRANCISCHINI; FURTADO; GARCIA, 2015). Nessa mesma vertente, a Teoria das Operações Internacionais afirma que as empresas devem utilizar os meios que possibilitem menor custo para elas, procurando uma integração tanto vertical como horizontal e escolhendo a melhor forma de entrada para o mercado estrangeiro, seja por meio de investimento estrangeiro direto, licenciamento ou exportação (IZIDORO; SANTOS, 2016). 
A Teoria do Paradigma Eclético, assim como a Teoria do Ciclo do Produto e a das Operações Internacionais, também se preocupa com fatores econômicos, afirmando que há uma integração entre aspectos de produção, investimento e comércio e se houver vantagem competitiva sobre os concorrentes, inicia-se a inserção no mercado exterior, sabendo que essa será a melhor escolha para a firma principal (SILVA et al., 2015).

Já para a Teoria da Distância Psíquica e a Interação Comprador-vendedor, um fator imprescindivel para o sucesso dos negócios é a flexibilidade para adaptar-se, pois isso impactará diretamente na vantagem competitiva das empresas e também diminuirá a distância psicológica que surge no processo de internacionalização, sendo pelas culturas diferentes, idiomas e até mesmo a interação e confiança dos relacionamentos (GERVASONI, 2013). O Modelo de Inovação também afirma que é preciso adequar-se em um cenário global onde há constantes mudanças tecnológicas, pois atualmente as empresas estão recrutando talentos de diversas partes do globo, além de terceirizar tecnologia da informação, softwares e principalmente a inovação (IZIDORO; BATISTA, 2016).

A Teoria da Escola de Uppsala, com sua vertente comportamental, afirma que as empresas visam um constante aprendizado por meio de experiências internacionais e o comprometimento com outras empresas estrangeiras, pois uma organização que passa por diferentes fases agrega adequações e crescimento para si mesma (PEREIRA; GOMES, 2017). Essa teoria ainda busca englobar os conceitos de redes de relacionamentos, pois acredita que o estabelecimento de relacionamentos internacionais, tanto pessoais como de negócios, influencia na escolha de novas estratégias e entradas para este mercado (GALDINO; COSTA, 2015).

Para Porto (2013), o processo de internacionalização não é simples e deve estar integrado com os objetivos estratégicos de uma organização, pois engloba atitude competitiva, ferramentas eficazes de administração, gestão do conhecimento e de relacionamentos entre clientes, fornecedores, parceiros e entre outros, além da capacidade de adaptação entre os contextos diferentes (econômicos, culturais e sociais). Por isso, diferentes teorias e diferentes modelos buscam explicar a internacionalização nas empresas; vale também ressaltar que não são apenas as empresas tradicionais que buscam expandir seus mercados (PORTO, 2013).

As universidades, por exemplo, com o fenômeno da globalização, também têm expandido seus mercados e, de acordo com Scherer (2015), a internacionalização das universidades pode ser entendida como uma rede de conexões, firmada por acordos entre universidade, trazendo diversos benefícios como a troca de experiência entre novos padrões de ensino, melhoria de metodologias e quebra de barreiras no acesso a experiências internacionais na educação superior. 


\subsection{A INTERNACIONALIZAÇÃO DAS UNIVERSIDADES}

O ensino superior, com os fatores da globalização e avanço tecnológico, tem sua expansão pautada em aspectos como a democratização e a internacionalização, pois, no atual contexto, não há mais espaço para formação de profissionais e cidadãos com foco na inserção exclusivamente local, já que as experiências contemporâneas (sejam pessoais ou profissionais) exigem o desenvolvimento de competências interculturais para o enfrentamento de desafios globais (MOROSINI; USTÁRROZ, 2016).

Para Dafouz e Smit (2014 apud DIDIO, 2018), o aspecto global dentro das universidades sempre se fez presente, porém, nos últimos 20 anos, observa-se um grande aumento no intercâmbio de alunos de diferentes regiões e até mesmo de diferentes países, gerando um contexto cada vez mais heterogêneo e com significativas diferenças linguísticas, culturais e acadêmicas. Leal e Moraes (2017) corroboram com os autores afirmando que, desde os anos 1990, têm-se observado uma crescente importância dada ao conhecimento com fins de desenvolver a economia e elevar a competitividade global, dessa forma, a internacionalização nas universidades ganha cada vez mais espaço no planejamento estratégico das instituições de ensino superior.

Segundo Prolo et al. (2019), a internacionalização tem como propósito criar condições que favorecem a instalação de processos de modernização, inovação, competitividade e inserção internacional no País. Assim, o governo busca, através deste mecanismo, integrar universidades, pesquisadores e estudantes à comunidade científica global.

De acordo com Bezerra (2017), os blocos econômicos têm ajudado nesse processo de internacionalização das universidades, o MERCOSUL (Mercado Comum do Sul), por exemplo, bloco econômico fundado por Brasil, Argentina, Uruguai e Paraguai, tem presenciado crescimento constante provindo da internacionalização. Para a autora, um exemplo disso é o fluxo cada vez mais intenso de alunos e professores entre as universidades dos países membros sendo que, além do processo bilíngue, também há um desenvolvimento científico e cultural, rompendo barreiras e contribuindo para a diminuição das desigualdades regionais.

No Brasil, o assunto sobre internacionalização no ensino superior passa a ser ainda mais debatido a partir de 2008, quando os Estados Unidos enfrentam efeitos negativos no orçamento de suas universidades por conta da grande recessão econômica (SPEARS, 2014). Para o autor, o intercâmbio entre universidades surgiu como um plano estratégico nacional bilateral, a fim de estabelecer uma parceria educacional entre os países, o que, posteriormente, incentivou o programa Ciência sem Fronteiras (atualmente nomeado Programa Brasileiro de Mobilidade Científica). 
Porém, Chaves e Castro (2016) ressaltam que o Brasil se introduz no processo de internacionalização deformatardia, poistem umsistema deensino superior jovem comparadoa outros sistemas, principalmente de países desenvolvidos. Os autores ainda argumentam que o país não possui universidades atrativas para o público estrangeiro, além da dificuldade do idioma português e também a falta de hospitalidade para com estudantes provindos do exterior. Finardi, Santos e Guimarães (2016) também corroboram quando afirmam que o Brasil é o $13^{\circ}$ país com maior produção acadêmica no mundo, porém, tal produção raramente tem impacto em cenários internacionais, levando em conta fatores linguísticos e culturais que impedem a circulação internacional de pesquisas brasileiras, ainda que robustas.

Com a finalidade de impulsionar a internacionalização, Morosini e Corte (2018) trazem algumas estratégias que devem ser adotadas pelas universidade brasileiras, como, por exemplo, a criação de um escritório internacional voltado especificamente para o processo de internacionalização da instituição; a formação de redes regionais, continentais e intercontinentais; a promoção de atividades de intercâmbio, congressos e demais eventos no exterior; a construção e difusão do conhecimento sobre redes e programas de cooperação; além de oferecer oportunidades para realização de mobilidades acadêmicas.

Além disso, o país também deve investir em tecnologia, pois é por meio dos avanços em tecnologia da informação que há maior fortalecimento dos sistemas educacionais, transmitindo dados importantes em um curto espaço de tempo entre um grande espaço territorial (BEZERRA, 2017). Para a autora, a educação é um bem público global onde todos devem ter direito e acesso de forma igualitária, por isso, um processo de internacionalização é de extrema importância a fim de consolidar bases solidárias e respeitosas com as diferentes culturas e povos.

Decorrente dessa crescente preocupação com a internacionalização de empresas, inclusive nas universidades, entende-se que é relevante compreender se esta necessidade também se reflete nas publicações, assim, o próximo capítulo delimita os processos metodológicos utilizados no estudo.

\section{METODOLOGIA}

O presente estudo caracteriza-se como uma investigação de abordagem quantitativa, de natureza descritiva, e foi desenvolvido a partir de uma pesquisa bibliométrica. A pesquisa referente às temáticas internacionalização e universidades foi realizada na base de dados Web of Science, considerando a totalidade dos artigos científicos publicados.

De acordo com Araújo (2006, p. 12) a bibliometria é caracterizada como sendo uma "técnica quantitativa e estatística de medição dos índices de produção e disseminação do conhecimento científico". Já a abordagem quantitativa, para Malhotra (2012), não se preocupa em apenas quantificar os dados, mas 


\section{Gestãoe \\ Desenvolvimento}

e-ISSN: 2446-6875

p-ISSN: $1807-5436$

também em aplicar formas de análise estatísticas a fim de medir informações sobre um determinado tema.

Ademais, a pesquisa pode ser classificada como descritiva, a qual tem como objetivo principal a descrição das características de determinada população, fenômeno ou ainda o estabelecimento de relações entre variáveis. Uma de suas características mais significativas está na utilização de técnicas padronizadas de coletas de dados (GIL, 2018).

\subsection{DEFINIÇÃO DO ESCOPO DO ESTUDO}

A realização da pesquisa dividiu-se em três etapas. Inicialmente realizou-se a busca com os termos "internationalization"e "university" no campo de pesquisa da base Web of Science. A seguir foram levantadas as características relevantes para a realização do presente estudo.

Em seguida, realizou-se a seleção dos hot topics e a análise dos artigos mais citados em comparação com os autores com mais publicações nesta temática. A seguir, a Figura 1 indica as etapas que foram seguidas durante a pesquisa.

Figura 1: Etapas da Pesquisa

Etapa 1: Pesquisar os termos internationalization (internacionalização) e university (universidade) na base principal da Web of Science.

Etapa 2: Pesquisar total de publicações, os autores com maior número de publicações, áreas temáticas, título das fontes, ano das publicações, instituições, agências de -financiamento, idiomas e países.

Etapa 3: Identificar hot-topics, número de publicações, índice h-b, índice m, combinar os termos com internationalization e university e analisar o número de publicações por autor e vezes que suas publicações foram citadas.

\section{Fonte: Elaborado pelos autores, 2019.}

Foram encontradas 2.272 publicações a respeito das temáticas internacionalização e universidade(s), buscando em todos os períodos disponiveis pela Web of Science (1945-2019). Porém, encontraram-se publicações apenas a partir de 1989 e, como as publicações do ano de 2019 ainda não estavam completas, dada a data de fechamento desta pesquisa, optou-se por analisar os anos de 1989 a 2018, totalizando 2.126 publicações. É importante salientar que a pesquisa foi realizada no mês de agosto de 2019. 


\section{Gestãoe \\ Desenvolvimento}

e-ISSN: 2446-6875

p-ISSN: $1807-5436$

\subsection{IDENTIFICAÇÃO DOS HOT TOPICS}

Com a finalidade de identificar os hot topics referentes aos temas da pesquisa, foram analisados os índices h-b e m. Hirsch (2005) propõe o índice h-index (índice-h) tendo em vista quantificar o impacto e a relevância da produção científica individual para a avaliação de pesquisadores e com isso realizar uma comparação de propósitos de pesquisa.

Banks (2006) apresenta o índice h-b como uma extensão do h-index, o qual é obtido por meio do número de citações de um tópico ou combinação em determinado período, listados em ordem decrescente de citações. $\mathrm{O}$ índice h-b é encontrado em publicações que tenham obtido um número de citações igual ou maior à sua posição no ranking.

O autor citado acima, também explica o cálculo do índice m, o qual é obtido pela divisão do índice $h$ - $b$ pelo período de anos que se deseja obter informações ( $n$ ). Para a análise dos índices $h-b$ e $m$, foram utilizadas as definições evidenciadas no Quadro 1.

\section{Quadro 1 - Definições para classificação de hot topics}

\begin{tabular}{|l|l|}
\hline \multicolumn{1}{|c|}{ Índice $\mathbf{m}$} & \multicolumn{1}{c|}{ Tópico/combinação } \\
\hline $0<\mathrm{m} \leq 0,5$ & $\begin{array}{l}\text { Pode ser de interesse para pesquisadores em um campo específico } \\
\text { de pesquisa, o qual engloba uma comunidade pequena. }\end{array}$ \\
\hline $0,5<\mathrm{m} \leq 2$ & $\begin{array}{l}\text { Provavelmente pode se tornar um hot topiccomo área de pesquisa, } \\
\text { no qual a comunidade é muito grande ou o tópico/combinação } \\
\text { apresenta características muito interessantes. }\end{array}$ \\
\hline $\mathrm{m} \geq 2$ & $\begin{array}{l}\text { É considerado um hot topic, tema exclusivo com alcance não } \\
\text { apenas na sua própria área de pesquisa e é provável que tenha } \\
\text { efeitos de aplicação ou características únicas. }\end{array}$ \\
\hline
\end{tabular}

\section{Fonte: Banks (2006).}

Com base nas definições propostas por Banks (2006), serão considerados hot topics as combinações com índice $m \geq 2$. Já as combinações que obtiverem um índice $m \geq 2$ serão considerados tópicos emergentes na área pesquisada.

É apresentada a seguir a discussão dos resultados da pesquisa a partir da bibliometria realizada. 


\section{ANÁLISE E DISCUSSÃO DOS RESULTADOS}

Os resultados da pesquisa evidenciaram as principais características da produção científica relacionados com os termos internationalizatione university, após a pesquisa dos termos na base de dados. A partir das definições do escopo do estudo, foram selecionadas 2.126 publicações, filtrando apenas por artigos científicos. Diante disso será realizada a análise dos dados, nas categorias definidas.

Inicialmente, selecionaram-se as 10 áreas do conhecimento que possuem mais publicações a respeito da temática, apresentadas na Tabela 1.

Tabela 1 - Áreas temáticas no estudo de internacionalização e universidade
\begin{tabular}{|ll|}
\hline \multicolumn{1}{|c|}{ Áreas Temáticas } & Número De Publicações \\
Education Educational Research & 1.157 \\
\hline Management & 193 \\
\hline Social Sciences Interdisciplinary & 182 \\
\hline Education Scientific Disciplines & 135 \\
\hline Business & 119 \\
\hline Linguistics & 114 \\
\hline Language Linguistics & 95 \\
\hline Economics & 75 \\
\hline Information Science Library Science & 67 \\
\hline Computer Science Interdisciplinary Applications & 64 \\
\hline
\end{tabular}

Fonte: Web of Science (2019).

Quanto aos periódicos que mais publicaram sobre o assunto no período estudado destacam-se: Journal of Studies in International Education (98), Inted Proceedings (95), Higher Education (79), Edulearn Proceedings (63), Advances in Social Science Education and Humanities Research (59), Procedia Social and Behavioral Sciences (41), Iceri Proceedings (32), Studies in Higher Education (25) e Scientometrics (24).

O periódico Journal of Studies in International Education que teve o maior número de publicações em relação a temática estudada tem como foco aspectos relacionados a internacionalização no ensino superior que contribuam para a; globalização; cooperação internacional e concorrência, capacitação e assistência ao desenvolvimento; políticas e práticas nacionais, regionais, transnacionais e transfronteiriças; políticas e práticas de garantia de qualidade; formulação de políticas públicas e institucionais e impacto; gestão 


\section{Gestãoe \\ Desenvolvimento}

e-ISSN: 2446-6875

p-ISSN: 1807-5436

institucional estratégica; avanços e uso de tecnologia; desenvolvimento docente e pessoal; inovação na mobilidade de estudantes e funcionários; e questões estudantis

Percebe-se ainda que os demais periódicos que contemplam trabalhos publicados envolvendo internacionalização e universidades, tem foco em diferentes áreas como: educação, tecnologia, gestão educacional, desenvolvimento, globalização, comportamento, ensino superior, entre outros.

A respeito dos autores que mais publicam, foram listados os 12 pesquisadores com maior quantidade de publicação na área, conforme é apresentado na Tabela 2.

Tabela 2 - Autores com maior número de publicações sobre internacionalização e universidade
\begin{tabular}{|ll|}
\hline \multicolumn{1}{c}{ Autores } & Número De Publicações \\
\hline Horta, H. & 6 \\
\hline Lasagabaster, D. & 6 \\
\hline Moore, E. & 6 \\
\hline Smith, I. & 6 \\
\hline Zhang, H. & 5 \\
\hline Aguilar, M. & 5 \\
\hline Chang, D. F. & 5 \\
\hline Engels, T. C. E. & 5 \\
\hline Kwiek, M. & 5 \\
\hline Sidhu, R. & 5 \\
\hline Volet, S. & 5 \\
\hline Yang, R. & 5 \\
\hline Yemini, M. & Fonte: Web of Science (2019). \\
\hline
\end{tabular}

O autor que mais se destacou foi Hugo Horta com um total de 10 publicações, seguido dos demais autores com 6 e 5 publicações no referido tema. Horta, H. éprofessor assistentena Divisão de Administração de Políticas e Educação em Ciências Sociais da Faculdade de Educação da Universidade de Hong Kong. Ele trabalha com temas voltados para a diversidade do ensino superior, política científica e financiamento, produtividade de pesquisa, networking, internacionalização do ensino superior e mobilidade acadêmica. 


\section{Gestãoe \\ Desenvolvimento}

e-ISSN: 2446-6875

p-ISSN: 1807-5436

Já no Gráfico 1, pode-se observar o número de publicações ao longo dos anos analisados.

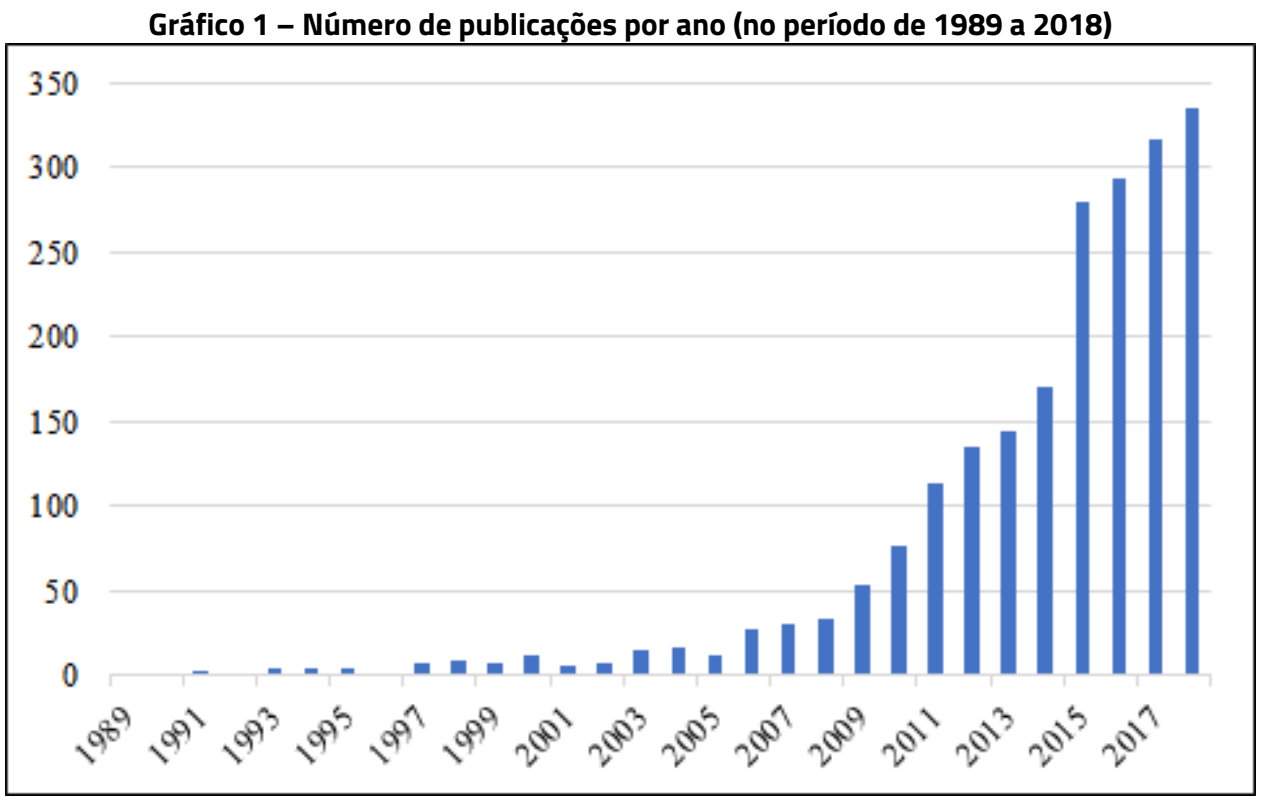

Fonte: Web of Science (2019).

Dado o histórico temporal das publicações a respeito dos termos internationalization e university, houve um aumento significativo a partir de 1989, sendo seu ápice, no ano de 2018 com 336 publicações, que representa 15,8\% em relação ao número total de artigos publicados. Com isso, existe um crescente interesse por parte dos pesquisadores, os quais tem investido em estudo em relação a temática internacionalização nas universidades.

Também foram levantadas as universidades com mais publicações, conforme Tabela 3. 


\section{Gestãoe \\ Desenvolvimento}

e-ISSN: 2446-6875

p-ISSN: $1807-5436$

Tabela 3 - Instituições com maior número de publicações sobre internacionalização e univer
\begin{tabular}{|ll|}
\multicolumn{1}{|c}{ Universidades } & Número De Publicações \\
\hline University of Hong Kong & 26 \\
\hline Tomsk Polytechnic University & 19 \\
\hline University of Nottingham & 17 \\
\hline Monash University & 16 \\
\hline University of British Columbia & 16 \\
\hline University of London & 16 \\
\hline University of South Australia & 15 \\
\hline Bucharest Academy of Economic Studies & 15 \\
\hline Complutense University of Madrid & 14 \\
\hline Education University of Hong Kong Eduhk & 14 \\
\hline Newcastle University Uk & 14 \\
\hline Universitat Politecnica De Valencia & 14 \\
\hline Ural Federal University & 14 \\
\hline
\end{tabular}

Fonte: Web of Science (2019).

Quanto às instituições que mais publicaram sobre a temática no período estudado, com seus respectivos países de origem, destacam-se: University of Hong Kong - China (26), Tomsk Polytechnic University - Rússia (19), University of Nottingham - Inglaterra (17), Monash University - Austrália (16 1, University of British Columbia - Canadá (16), University of London - Inglaterra (16), University of South Australia - Austrália (15), Bucharest Academy of Economic Studies - Romenia (14), Complutense University of Madrid - Espanha (14), Education University of Hong Kong Eduhk - China (14), Newcastle University Uk Inglaterra (14), Universitat Politecnica de Valencia - Espanha (14) e Ural Federal University - Rússia (14) .

Em relação as agências financiadoras, tem ênfase a Economic and Social Research Council com 8 publicações, European Commission, 8, Spanish Ministry of Economy and Competitiveness, 7, National Natural Science Foundation of China, 5, Australian Research Council, 4 e Spanish Ministry of Science and Innovation, 4. As demais instituições citadas na Web of Science tiveram no máximo 3 publicações cada. Ambas as agências que tem o maior número de publicações (8) estão localizadas no continente europeu, o qual investe de forma exacerbada em pesquisas fomentando a cientificidade de seus países.

Já com relação aos países que mais produzem, a China está em primeiro lugar com 347 publicações, os Estados Unidos com 222, Inglaterra com 208, Espanha com 195, Austrália, 161, Rússia, 112, Alemanha, 


\section{Gestãoe \\ Desenvolvimento}

e-ISSN: 2446-6875

p-ISSN: $1807-5436$

82, Canadá 67, Romênia com 59, Brasil 58, Japão, 49, Itália, 46, Suécia, 44, Polônia, 42, Portugal, 38, Malásia, 37, Países Baixos, 37, África do Sul, 36, Finlândia e Taiwan com 31, Dinamarca e Turquia, 29, Bélgica, 28, Coreia do Sul, 27 e França, 24. Estes dados são apresentados no Figura 2.

Figura 2 - Publicações por países

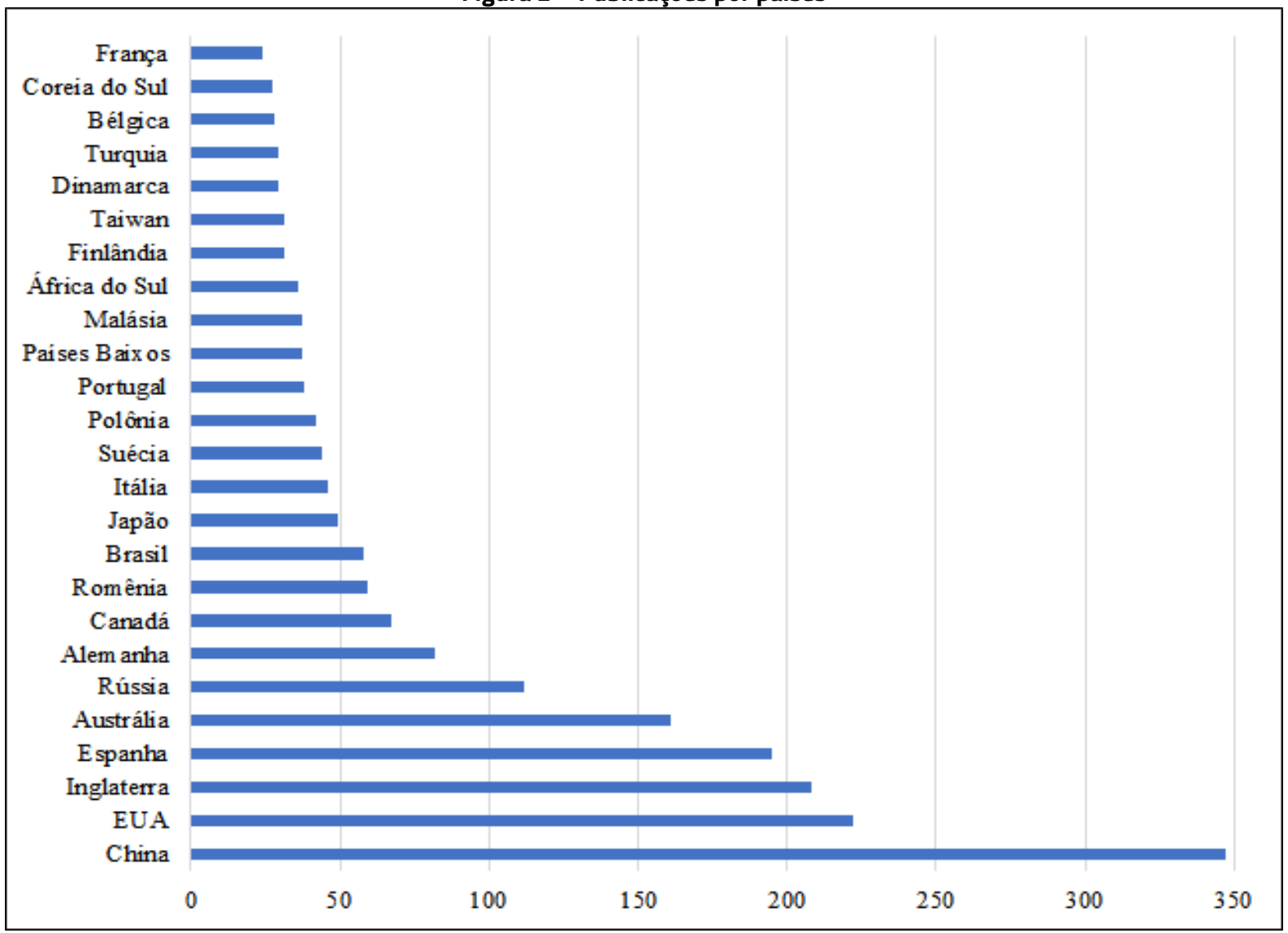

Fonte: Web of Science (2019).

Comparando as instituições que mais publicam e as agências financiadoras, nota-se que todas se encontram nos países com melhores colocações a respeito do número de publicações. Complementando, também foi percebido que o idioma principal é o inglês, com 1.921 publicações, seguido do espanhol com 77, português com 32, russo e chinês com 29, demostrando a importância da língua inglesa para a troca de experiências a nível mundial.

Após a etapa de verificar as principais características das publicações, foi realizada uma leitura dos resumos dos artigos mais citados para identificar os tópicos relevantes sobre internacionalização e 


\section{Gestãoe \\ Desenvolvimento}

e-ISSN: 2446-6875

p-ISSN: $1807-5436$

universidade. Dessa forma, foram definidos 11 tópicos que mais se repetiam nos resumos, os quais foram pesquisados individualmente na Web of Science no período de 1989 até 2018, conforme estabelecido para a realização do presente estudo. Os tópicos selecionados estão apresentados na Tabela 4.

Tabela 4 - Tópicos relacionados com as principais publicações sobre os temas internacionalização e universidade

\begin{tabular}{|ll|}
\hline \multicolumn{1}{|c|}{ Tópicos } & \multicolumn{1}{|c|}{$\begin{array}{l}\text { Número de } \\
\text { publicações }\end{array}$} \\
Development (Desenvolvimento) & 3.570 .983 \\
\hline Research (Pesquisa) & 2.983 .589 \\
\hline Science (Ciência) & 1.542 .021 \\
\hline Culture (Cultura) & 1.241 .143 \\
\hline Students (Estudantes) & 637.770 \\
\hline Markets (Mercados) & 624.579 \\
\hline Competition (Competição) & 285.975 \\
\hline Globalization (Globalização) & 49.739 \\
\hline Organizational Change (Mudança Organizacional) & 33.810 \\
\hline Academic Governance (Governança Acadêmica) & 3.968 \\
\hline Intercultural Experience (Experiência Intercultural) & 2.429 \\
\hline
\end{tabular}

Fonte: Web of Science (2019).

Na Tabela 5, citam-se os tópicos abordados na Tabela 4 combinados com os termos internationalization e university, no intervalo de 1989 a 2018. Dessa forma, foi identificado o índice h-b de cada um dos termos o que possibilitou a realização do cálculo do índice m. 


\section{Gestãoe \\ Desenvolvimento}

e-ISSN: 2446-6875

p-ISSN: 1807-5436

Tabela 5 - Internacionalização e universidade e seus possíveis hot-topics

\begin{tabular}{|c|c|c|c|}
\hline $\begin{array}{l}\text { Tópicos } \\
\text { Research (Pesquisa) }\end{array}$ & $\begin{array}{l}\text { Publicações } \\
1.033\end{array}$ & $\begin{array}{l}\text { Índice } \mathbf{h - b} \\
41\end{array}$ & $\begin{array}{l}\text { Índice } \mathbf{m} \\
1,41\end{array}$ \\
\hline Students (Estudantes) & 1.159 & 37 & 1,28 \\
\hline Development (Desenvolvimento) & 816 & 32 & 1,10 \\
\hline Globalization (Globalização) & 455 & 31 & 1,07 \\
\hline Science (Ciência) & 343 & 23 & 0,79 \\
\hline Markets (Mercados) & 319 & 22 & 0,76 \\
\hline Culture (Cultura) & 280 & 20 & 0,69 \\
\hline $\begin{array}{l}\text { Intercultural Experience (Experiência } \\
\text { Intercultural) }\end{array}$ & 93 & 17 & 0,59 \\
\hline $\begin{array}{l}\text { Organizational Change (Mudança } \\
\text { Organizacional) }\end{array}$ & 33 & 9 & 0,31 \\
\hline $\begin{array}{l}\text { Academic Governance (Governança } \\
\text { Acadêmica) }\end{array}$ & 25 & 7 & 0,24 \\
\hline
\end{tabular}

Fonte: Web of Science (2019).

Observa-se que nenhum tópico se torna um hot-topic $(\mathrm{m} \geq 2)$ nos anos de publicação a respeito da temática, porém, isso pode acontecer por conta do grande período de anos analisado na presente pesquisa, onde nos primeiros anos, e até mesmo nos anos atuais, ainda existe uma carência de estudos sobre internacionalização de universidades se comparado a outros temas como sustentabilidade, desenvolvimento, administração e, até mesmo, internacionalização de empresas.

Mesmo assim, optou-se por analisar, os tópicos que são considerados emergentes ( $m>0,5)$, os dez autores com maior número de publicações. Ademais, foi verificado, dentre esses pesquisadores, quais aparecem como autores das dez publicações mais citadas para cada combinação, conforme apresentado na Tabela 6. 


\section{Gestãoe \\ Desenvolvimento}

e-ISSN: 2446-6875

p-ISSN: $1807-5436$

Tabela 6 - Relação entre os autores com mais publicações e publicações mais citadas

\begin{tabular}{|c|c|c|}
\hline $\begin{array}{c}\text { TópIcos } \\
\text { Research (Pesquisa) }\end{array}$ & $\begin{array}{l}\text { AUTORES COM MAIS PUBLICAÇõES } \\
\text { HORTA, H. (7)'; KWIEK, M. (6); YEMINI, M. (4); } \\
\text { BAGCHI-SEM, S. (3); BEDENLIER, S. (3); DAFOUZ, } \\
\text { E. (3); DEL CAMPO, C. (3); HARRIS, N. (3); HEALEY. } \\
\text { N. M. (3); HEITOR, M. (3). }\end{array}$ & PUBLICAÇÕES MAIS CITADAS \\
\hline Students (Estudantes) & $\begin{array}{l}\text { SMITH, I. (6); ZHANG, H. (6); } \\
\text { AGUILAR, M. (5); DEL CAMPO, C. (4); DOIZ, A. } \\
\text { (4); HARRISON, N. (4); LASAGABASTER, D. (4); } \\
\text { NEAGU, A. M. (4); SIDHU, R. (4); STEIN, S. (4). }\end{array}$ & $\begin{array}{l}\text { 10. }{ }^{2} \text { HARRISON, N.; PEACOCK, N. Cultural distance, mindfulness } \\
\text { and passive xenophobia: using Integrated Threat Theory to } \\
\text { explore home higher education students' perspectives on } \\
\text { 'internationalisation at home.' British Educational Research } \\
\text { Journal, v. } 36, \text { n. } 6,2010(67)^{3} \text {. }\end{array}$ \\
\hline $\begin{array}{l}\text { Development } \\
\text { (Desenvolvimento) }\end{array}$ & $\begin{array}{l}\text { HORTA, H. (6); LO, W. Y. W. (4); BAGCHI- } \\
\text { SEM, S. (3); BELIAEVA, V. S. (3); HEALEY, N. M. } \\
\text { (3); LAPINA, I. (3); LEASK, B. (3); NYSTROM, T. (3); } \\
\text { POPESCU, F. (3); SMITH, H. L. (3). }\end{array}$ & $\begin{array}{l}\text { 4. KEEBLE, D.; LAWSON, C.; SMITH, H. L.; MOORE, B.; } \\
\text { WILKINSON, F. Internationalisation processes, networking } \\
\text { and local embeddedness in technology-intensive small firms. } \\
\text { Small Business Economics, v. } 11, \mathrm{n} .4,1998 \text { (116). }\end{array}$ \\
\hline $\begin{array}{l}\text { Globalization } \\
\text { (Globalização) }\end{array}$ & $\begin{array}{l}\text { YANG, R. (5); KLIMOVA, B. (3); MARGINSON, S. } \\
\text { (3); SIDHU, R. (3); YEMINI, M. (3); } \\
\text { BOURN, D. (2); BUFREM, L. S. (2); } \\
\text { CANIGLIA, G. (2); CHANG, D. F. (2); CHARLES, H. (2). }\end{array}$ & $\begin{array}{l}\text { 1. MARGINSON, S. Dynamics of national and global } \\
\text { competition in higher education. Higher Education, v. 52, n. 1, } \\
2006 \text { (398). }\end{array}$ \\
\hline Science (Ciência) & 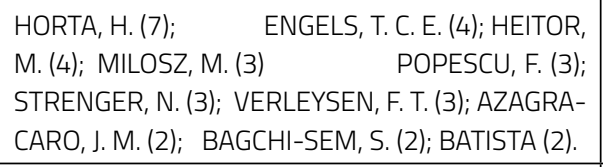 & - \\
\hline Markets (Mercados) & 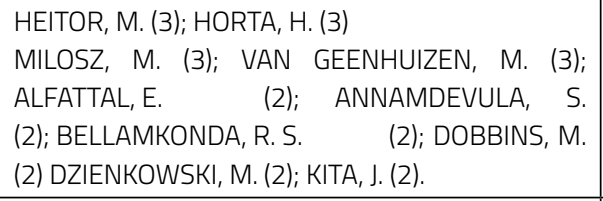 & - \\
\hline Culture (Cultura) & $\begin{array}{l}\text { TRUYEN, F. (3); DUNNE, C. (2); FOZDAR, F. (2); } \\
\text { GOODMAN, L. (2); HERBERTSON, N. (2); KUPPENS, } \\
\text { A. (2); LEMOINE, J. (2); LIU, Z. (2); MARKOVIC, L. (2); } \\
\text { PROSS, E. (2). }\end{array}$ & $\begin{array}{l}\text { 4. DUNNE, C. Host Students' Perspectives of Intercultural } \\
\text { Contact in an Irish University. Journal of Studies in } \\
\text { International Education, v. } 13, \text { n. } 2,2009(56) \text {. }\end{array}$ \\
\hline $\begin{array}{l}\text { Intercultural Experience } \\
\text { (Experiência Intercultural) }\end{array}$ & 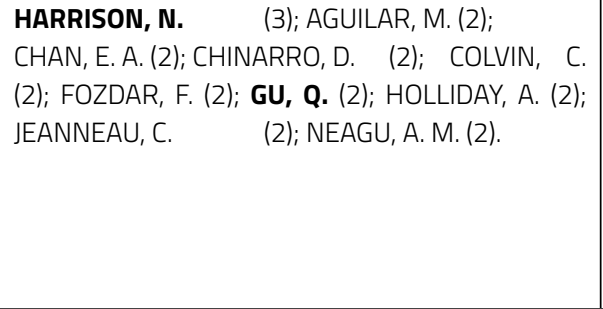 & $\begin{array}{l}\text { 1. GU, Q.; SCHWEISFURTH, M.; DAY, C. Learning and growing } \\
\text { in a 'foreign' context: intercultural experiences of international } \\
\text { students. Compare-A Journal of Comparative and } \\
\text { International Education, v. 40, n. 1, } 2010 \text { (120). } \\
\text { 6. PEACOCK, N.; HARRISON, N. "It's So Much Easier to Go With } \\
\text { What's Easy" "Mindfulness" and the Discourse Between Home } \\
\text { and International Students in the United Kingdom. Journal of } \\
\text { Studies in International Education, v. 13, n. 4, } 2009 \text { (45). }\end{array}$ \\
\hline
\end{tabular}

${ }^{1}$ Número de publicações de cada autor sobre o tópico;

2 Posição da publicação em relação ao número de citações recebidas;

${ }^{3}$ Número de vezes que a publicação foi citada.

Fonte: Web of Science (2019). 
A partir dos dados apresentados, pode-se observar que o número de publicações por autor não está diretamente relacionado com o impacto das mesmas, pois apenas os autores HARRISON, N (studentes e intercultural experience), SMITH, H. L. (developmente), MARGINSON, S. (globalization), DUNNE, C. (culture) e por fim, GU, Q. (intercultural experience) apareceram como os autores que mais publicam acerca da temática relacionadas com os respectivos tópicos.

Os autores que mais publicaram sobre a temática conforme constam na Tabela (2), também estão identificados na Tabela (6) como sendo os autores que mais publicam no que diz respeito a combinação do termo internationalization e university com os tópicos considerados emergentes. Diante disso, foram identificados os tópicos que os autores mais pesquisam: Horta, H. (research, development, sciencee markets), LASAGABASTER, D., SMITH, I. (students e development), ZHANG, H. (students), AGUILAR, M. (students e intercultural experience), CHANG, D. F. (globalization), ENGELS, T. C. E. (science), KWIEK, M. (research), SIDHU, R. (students e globalization), YANG, R. (globalization), YEMINI, M. (research e globalization).

Ademais, MOORE, E. e SMITH, I. são autores da publicação mais citada no que compete a temática internacionalização e universidade no tópico desenvolvimento (development) com o artigo intitulado: Internationalisation processes, networking and local embeddedness in technology-intensive small firms.

\section{CONSIDERAÇÕES FINAIS}

O tema internacionalização e universidade constitui um contexto que vem ganhando cada vez mais abrangência no âmbito mundial, tendo em vista promover a diversidade dentro do ambiente acadêmico. O processo de internacionalização das universidades, além de possibilitar a integração de seu corpo acadêmico à comunidade científica global, favorece a instalação de processos de modernização, inovação, competitividade e inserção internacional no País.

Evidencia-se um progressivo crescimento desde o surgimento de estudos no ano de 1989, chegando ao seu ápice no ano de 2017. Porém, no último ano analisado, no caso 2018, ocorreu uma significativa diminuição acerca das pesquisas que relacionam ambas as temáticas.

Diante do considerável aumento do número de publicações dos últimos anos e da relevância do tema proposto, vem despertando cada vez mais interesse por parte dos pesquisadores em diversas discussões a nível mundial, nas mais diversas áreas, em especial educação, gestão, ciências sociais, linguística e economia. Pode-se concluir que existe uma multidisciplinariedade de campos de estudo, que evidencia uma diversidade de interpretações, no que diz respeito a abordagens, conceitos e definições acerca da temática. 
Em relação ao veículo de divulgação, verificou-se uma representatividade na publicação de artigos, em que o Journal of Studies in International Education teve maior destaque com 99 publicações, com foco em publicações com foco na internacionalização do ensino superior por meio da relação com diversos aspectos como a globalização. Também destacaram-se o Journal Inted Proceedings e Higher Education, que publicaram 95 e 80 artigos respectivamente durante o período estudado.

Quanto aos países que ocupam o topo em número de publicações estão a China, Estados Unidos, Inglaterra e Espanha. A China é o país que se destaca em relação ao incentivo ao desenvolvimento de pesquisas, sendo comprovado neste estudo através do grande número de publicações da University of Hong Kong. Ainda, o autor que destacou-se Horta, H., atualmente é professor na área de Administração e Educação da mesma instituição.

Além disso, não foram encontrados temas que pudessem ser classificados como hot-topics, isso se deve ao fato de a pesquisa ter sido realizada desde os anos em que começaram a serem desenvolvidos estudos sobre a temática em questão. Porém, optou-se por analisar os tópicos considerados emergentes, como: research(pesquisa), students(estudantes), development(desenvolvimento), globalization (globalização), science (ciência), markets (mercados), culture (cultura) e intercultural experience (experiência intercultural).

Por fim, através das informações apresentadas na Tabela 6, pode-se concluir que o número de publicações por autor não está diretamente relacionado com as publicações que são mais citadas, pois da lista dos pesquisadores que mais publicam acerca da temática conforme consta na Tabela 2, apenas Moore e Smith estão na lista dos das publicações mais citadas no período estudado.

Percebe-se que as publicações que relacionam o tema vêm aumentando de forma gradativa na última década, sendo que o número de estudos está crescendo em um ritmo acelerado nas diferentes áreas do conhecimento, porém ainda consiste em um tema emergente e pouco explorado, com amplo campo para o desenvolvimento de novas pesquisas.

A contribuição deste estudo para os estudos em gestão, se deve aos indicadores resultantes quanto aos autores, às instituições de pesquisa e aos periódicos que mais se destacam na produção do conhecimento sobre o tema em nivel internacional, com o intuito de expandir os conhecimentos no que se refere a temática. Já para a academia e para a sociedade, em geral, este estudo contribui na perspectiva de fomentar novos estudos e possibilitar o acesso facilitado aos autores e artigos mais citados e referências na temática.

A principal limitação deste estudo refere-se ao fato de que as informações apresentadas estão limitadas aos artigos encontrados na base Web of Science. Para estudos futuros, sugere-se ampliar a busca dos temas internacionalização e universidades em outras bases de dados, bem como em eventos científicos a nivel nacional e internacional, a fim de obter maiores informações a respeito do tema. 


\section{Gestãoe \\ Desenvolvimento}

e-ISSN: 2446-6875

p-ISSN: $1807-5436$

\section{REFERÊNCIAS}

ARAÚJO, C. A. A. Bibliometria: evolução histórica e questões atuais. Em Questão, v. 12, n. 1, p. 11-32, 2006.

BANKS, M. G. An extension of the Hirsch index: indexing scientific topics and compounds. Scientometrics, Budapeste, Hungria, v. 69, n. 1, p. 161-168, 2006.

BATISTA, M. L. C.; IZIDORO, C. Uma análise do processo de internacionalização de uma empresa de tecnologia da informação. Actus, v. 1, n. 1, 2016.

BEZERRA, A. F. D. Internacionalização da educação superior no mercosul: uma análise comparada de universidades públicas de Argentina e Brasil. In: Anais Eletrônicos. XVII Colóquio Internacional de Gestão Universitária, Mar del Plata, 2017.

CHAVES, V. L. J.; CASTRO, A. M. D. A. Internacionalização da educação superior no Brasil: programas de indução à mobilidade estudantil. Revista Internacional de Educação Superior, v. 2, n. 1, 2016.

DIDIO, A. R. Políticas linguísticas voltadas para a internacionalização do ensino superior: uma descrição do cenário atual da UFRGS. Trabalho de Conclusão de Curso. UFRGS, Porto Alegre, 2018.

FINARDI, K. R.; PORCINO; M. C. Tecnologia e metodologia no ensino de inglês: impactos da globalização e da internacionalização. Ilha Desterro, n. 66, 2014.

FINARDI, K.; SANTOS, J.; GUIMARÃES, F. A relação entre línguas estrangeiras e o processo de internacionalização: evidências da Coordenação de Letramento Internacional de uma Universidade Federal. Interfaces Brasil/Canadá, Revista Brasileira de Estudos Canadenses, v. 16, n. 1, 2016.

FRANCISCHINI, A. S. N.; FURTADO, J.; GARCIA, R. Tecnologia e trajetórias de internacionalização precoce: análise de casos na indústria brasileira. Gestão \& Produção, v. 22, n. 2, 2015.

GALDINO, C. B.; COSTA, L. F. L. G. Internacionalização de empresas exportadoras: aspectos motivacionais e estratégicos. Holos, v. 6, n. 31, 2015.

GERVASONI, V. C. Distância psíquica no processo de internacionalização: a percepção dos expatriados brasileiros. Dissertação de Mestrado. USCS, São Caetano do Sul, 2013.

GIL, A. C. Como elaborar projetos de pesquisa. 6 ed. São Paulo: Atlas, 2018.

HIRSCH, J. E. An index to quantify an individual's scientific research output. Proceedings of the National Academy of Sciences of the United States of America, v. 102, p. 16569-16572, 2005. 


\section{Gestãoe \\ Desenvolvimento}

e-ISSN: 2446-6875

p-ISSN: $1807-5436$

IZIDORO, C.; SANTOS, A. C. C. S. Uma análise dos principais meios de entrada utilizados pelo grupo Gerdau no processo de internacionalização. Actus, v. 1, n. 1, 2016.

LEAL, F. G.; MORAES, M. C. B. Perspectivas de atuação do secretário executivo na gestão da internacionalização da educação superior. Revista de Gestão e Secretariado - GeSec, v. 8, n. 1, 2017.

MALHOTRA, N. K. Pesquisa de marketing: uma orientação aplicada. 6. ed. Porto Alegre: Bookman, 2012.

MARTINS, A.; REIS; E. C. Internacionalização na UFSC: análise do programa inglês sem fronteiras e do curso extracurricular de inglês. In: Anais Eletrônicos. XV Colóquio Internacional de Gestão Universitária, Mar del Plata, 2015.

MOROSINI, M. C.; CORTE, M. G. D. Teses e realidades no contexto da internacionalização da educação superior no Brasil. Revista Educação em Questão, v. 56, n. 47, 2018.

MOROSINI, M.; USTÁRROZ, E. Impactos da internacionalização da educação superior na docência universitária: construindo a cidadania global por meio do currículo globalizado e das competências interculturais. Em Aberto, v. 29, n. 97, 2016.

PEREIRA, A. J. P.; GOMES, J. S. Um estudo das estratégias de internacionalização das indústrias farmacêuticas brasileiras. Revista de Contabilidade e Organizações, v. 11, n. 29, 2017.

PORTO, J. V. J. 0 processo de internacionalização de empresas exportadoras da AMREC: um estudo multicaso com enfoque nos desafios e oportunidades. Monografia. UNESC, Criciúma, 2013.

PROLO, I; VIEIRA, R. C.; LIMA, M. C.; LEAL, F. G. Internacionalização das Universidades Brasileiras-Contribuições do Programa Ciência sem Fronteiras. Administração: Ensino e Pesquisa, v. 20, n. 2, p. 1-27, 2019.

PUZIOL, J. K. P.; BARREYRO, G. B. Internacionalização, regionalização, integração e a educação superior: algumas considerações sobre a acreditação regional do mercosul. Educação e Fronteiras On-Line, v. 6, ก. 16, 2016.

SANTIN, D. M.; VANZ, S. A. S.; STUMPF, I. R. C. Internacionalização da produção científica brasileira: políticas, estratégias e medidas de avaliação. RBPG, v. 13, n. 30, 2016.

SCHERER, B. O processo de internacionalização de universidades: um estudo de caso da UNIVATES. Monografia. UNIVATES, Lajeado, 2015. 


\section{Gestãoe \\ Desenvolvimento}

e-ISSN: 2446-6875

p-ISSN: $1807-5436$

SILVA, A. S. L.; LUNA, R. A.; ARRUDA, S. H.; FORTE, C.; MOURA, A. R. PIB e o fluxo das fusões e aquisições internacionais por empresas brasileiras. Qualitas Revista Eletrônica, v. 17, n. 1, 2016.

SOUZA, C. D.; FILIPPO, D.; CASADO, E. S. Impacto do Programa de Apoio a Planos de Reestruturação e Expansão das Universidades Federais Brasileiras (REUNI) na atividade investigativa: crescimento, qualidade e internacionalização. Em Questão, v. 21, n. 3, 2015.

SPEARS, E. O valor de um intercâmbio: mobilidade estudantil brasileira, bilateralismo \& internacionalização da educação. Revista Eletrônica de Educação, v. 8, n. 1, 2014.

VIEIRA, R. A. A. A internacionalização dos grupos hoteleiros portugueses: fatores que influenciam a escoIha do modo de entrada. Dissertação de Mestrado. UA, Aveiro, 2014. 